\title{
Tiques d'animaux sauvages en Haute-Volta
}

\author{
(Acariens, Ixodida)
}

par P. C. MOREL

\begin{abstract}
RÉSUMÉ
Vingt-quatre espèces de tiques ont été recensées sur les animaux sauvages de Haute-Volta. De 5 à 10 espéces pourront certainement s'ajouter à cette liste, après l'étude systématique du groupe d'Haemaphysalis leachi ou des récoltes de tiques sur éléphants, buffles, dans les nids d'oiseaux Ardéiformes ou dans les terriers de rongeurs. Dans leur ensemble, les espèces mentionnées de HauteVolta sont représentatives de la faune des savanes soudaniennes et soudanoguinéennes.
\end{abstract}

Depuis plus de 25 ans des entomologistes médicaux et vétérinaires travaillent en HauteVolta, dans des organismes situés notamment à Bobo-Dioulasso. En plus des groupes d'insectes qui constituent l'objet de leurs études, ils ont recueilli des tiques, méthodiquement ou au hasard de leurs déplacements, sur animaux domestiques ou sauvages.

Certaines données sur les espèces de tiques présentes dans le pays, leur distribution, leurs hôtes, en ont déjà été publiées (VILLIERS, 1955, MOREL, 1958 et 1961 ; MOREL et VASSILIADES, 1963 et 1965 ; LAMONTELLERIE, 1966).

Les récoltes se sont poursuivies depuis ces dates. Devant l'assez grand nombre de renseignements concernant les tiques d'animaux sauvages, reptiles, oiseaux ou mammifères, il a semblé intéressant d'en faire une revue générale, car il n'y a pas encore de publication sur ce sujet pour l'Ouest-Africain. Du fait de la position centrale de la Haute-Volta, on a ainsi une image de la faune des tiques parasites des vertébrés terrestres dans les savanes moyennes (soudaniennes et soudano-guinéennes) d'Afrique occidentale. Les résultats ou les lacunes dans les données seront commentés dans la conclusion.
Les collecteurs qui se sont succédé ou qui travaillent toujours sur place sont les suivants :

O.C.C.G.E.: Jacques HAMON, André RICKENBACH, Marcel EYRAUD, Max OVAZZA, Jean-Paul ADAM, Michel LAMONTELLERIE, Raymond SUBRA, Jacques BRENGUES, Claude LAVEISSIERE, Gérard DUVALLET, Jean-Paul HERVY, Bertrand SELLIN.

I.E.M.V.T.: Michel CLAIR, Dominique CUISANCE, Heinz POLITZAR, Yves TAZE.

Je remercie tous ces collègues qui m'ont confié ou m'ont permis d'examiner leurs collections, ou qui m'ont communiqué des listes de déterminations effectuées sur du matériel de Haute-Volta récolté par des collègues cités cidessus : je nomme plus particulièrement JeanLouis CAMICAS (ORSTOM, Dakar).

Les récoltes effectuées à la Léraba (Côted'Ivoire) sur la rive droite de la rivière du même nom, ont été citées car il s'agit d'une localité frontalière avec la Haute-Volta.

* Les récoltes déjà mentionnées dans nos publications sont désignées d'un astérisque. 
Quand il s'agit d'autres auteurs, leurs noms sont indiqués.

A titre de comparaison, on pourra se reporter à d'autres textes portant, en tout ou en partie, sur les tiques d'animaux sauvages dans les savanes d'Afrique occidentale et centrale.

Ce sont : ROUSSELOT (1951 et 1953 : Mali et Congo), VILLIERS (1955 : Afrique occidentale), MOREL (1956 et 1961: Niokolokoba, Sénégal, 1963: monts Nimba et Tonkoui, Côte-d'Ivoire), AESCHLIMANN (1967 : Côted'Ivoire), HOFFMANN et LINDAU (1971 : Niger), MOREL et MAGIMEL (1959: Tchad), MOREL et GRABER (1961 : Tchad), MOREL et FINELLE (1961: Centrafrique), HOOGSTRAAL (1956 : Soudan) MOREL et MOUCHET (1958 et 1965 : Cameroun), THAL (1972 : Centrafrique).

\section{Amblyomma nuttalli}

La Léraba :

Herpestes ichneumon $(1 \mathrm{~N}, \quad 28.03 .68$, O. C. C. G. E.)

Xerus erythropus $(1 \mathrm{~N}, 06.06 .75$, O. C. C. G. E.)

Thryonomys swinderianus (3NN, 06.1973, O. C. C. G. E.)

Kinixys belliana (7MM, 1F, 14.05.74, 2MM, 25.03.76 O. C. C. G. E.).

Logoniéké : Python regius (1M, 28.07.76, I. E. M, V. T.)

* Bobo-Dioulasso :

Varanus exanthematicus (3NN, 10.1950, O. C. C. G. E.)

Varanus sp. (3MM, 03.05.68, O. C. C. G. E.) Xerus erythropus (10NN, 8LL, 27.11.62, O. C. C. G. E.).

Atelerix albiventris (1M, 27.02.61; $2 \mathrm{MM}$, $15.07 .63 ; 1 \mathrm{~F}, 26.12 .74$, O. C. C. G. E., $1 \mathrm{M}, 27.07 .73$, I. E. M. V. T.).

Chien (1N, 15.10.76, I. E. M. V. T.).

Kadouba (Layassa) : Python sp. (1M, 11.11.71, O. C. C. G. E.).

Gombélédougou : Atelerix albiventris $(1 \mathrm{~N}$, 19.01.77, I. E. M. V. T.).

Nabéré (Gombélédougou) : Tryonomys swinderianus (1N, 23.03.74, O. C. C. G. E.).
Parasite habituel, à tous les stades, des tortues, varans, pythons et hérissons ; larves et nymphes possibles sur oiseaux (par exemple francolins et pintades) et sur mammifères.

Depuis les savanes soudaniennes jusqu'en forêt.

\section{Amblyomma tholloni}

Dankana (Batié) : homme (1F, dans une narine, 10.1971, O. C. C. G. E.).

Seule référence pour la Haute-Volta de l'Amblyomma spécifique de l'éléphant dans toute l'Afrique.

\section{Amblyomma variegatum}

La Léraba :

Xerus erythropus (IN, 06.06.75, O.C.C.G.E.).

Thryonomys swinderianus (1N, 17.02.74, O. C. C. G. E.).

Bobo-Dioulasso : Lepus crawshayi $(1 \mathrm{~N}, 18$. 02.74).

* Bobo-Dioulasso :

Francolinus bicalcaratus $(1 \mathrm{~N}, 09.07 .53,3 \mathrm{NN}$, $14.07 .57,6 \mathrm{NN}, 16.03 .74$; $\quad$ O. C. C. G. E.) Numida meleagris (2NN, 08.06.53, O. C. C. G. E.).

Varanus exanthematicus (2NN, 10.1950, O. C. C. G. E.).

Chamaeleo vulgaris (1N, 08.10.56, O. C. C. G. E.).

Komoé :

Sylvicapra grimmia $(1 \mathrm{~N}, 26.02 .77 ; 1 \mathrm{~N}$, 29.02 .77 ; I. E. M. V. T.).

Cephalophus rufilatus (7NN, 3LL, 25.02.77, I. E. M. V. T.).

Ourebia ourebi $(1 \mathrm{~N}, 25.01 .76,9 \mathrm{NN} ; 15.02$. 76, LL, 05.12.76 ; LL, 12.12.76, LL, 09.01.77 ; 24NN, 05.02.77;22NN, 25.02.77; I.E.M.V.T.).

Adenota kob (1N, 25.02.77, I. E. M. V. T.).

Alcelaphus major (11NN, 25.01.76;2NN, $8 \mathrm{LL}, 01.01 .77 ; 1 \mathrm{M}, 9 \mathrm{NN}, 05.02 .77 ; 2 \mathrm{NN}$, 23.01 .77 ; I. E. M. V. T.).

Hippotragus equinus (2NN, 25.02.77, I. E. M. V. T.).

Syncerus caffer (6LL, 02.01.77, I.E.M.V.T.). 
Phacochoerus aethiopicus (1N, 25.01.76; 21NN, 23.01.77 ; I. E. M. V. T.).

Guimpi (Kourinion) : Cercopithecus aethiops (2LL, 04.01.77, I. E. M. V. T.).

Sama : Ourebia ourebi (1N, 21.01.75, I. E. M. V. T.).

* Diébougou : Ourebia ourebi (2MM, 26.01.47, O. C. C. G. E.).

* Karankasso : Syncerus caffer (4MM, 05.1954, O. C. C. G. E.).

* Dougoumato (Kombia) : Sylvicapra grimmia (1M, 02.08.56, O. C. C. G. E.).

Koumbia :

Phacochoerus aethiopicus (1N, 03.1963, O. C. C. G. E.).

Thryonomys swinderianus (5NN, 03.1963, O. C. C. G. E.).

Gombélédougou :

Phacochoerus aethiopicus (5MM, 6FF, 18.05.59. O. C. C. G. E.).

Alcelaphus major (8NN, 02.02.75,

I. E. M. V. T.).

Kari (Houndé) :

Hippotragus equinus (1M, 03.03.74; $1 \mathrm{M}$, 19.07 .74 ; O. C. C. G. E.).

Canis aureus (7NN, 14.02.74, O. C. C. G. E.). Genetta tigrina (1N, 17.01.77, O. C. C. G. E.).

Francolinus bicalcaratus $(5 \mathrm{NN}, 17.01 .77$, O. C. C. G. E.).

Yakala (Garango) :

Lissotis melanogaster $(15 \mathrm{NN}, 07.11 .58$, O. C. C. G. E.).

* Ouagadougou : Eupodotis senegalensis (1N, 19.02.56, I. E. M. V. T.).

Dori: Phacochoerus aethiopicus (3NN, 07.02.62, O. C. C. G. E.).

Larves et nymphes ubiquistes sur tous vertébrés terrestres, adultes sur ongulés et carnivores, domestiques et sauvages.

Depuis le sud du Sahel jusque dans les clairières de forêt.

LAMONTELLERIE (1966) signale de plus en Haute-Volta comme hôtes des larves et des nymphes: Atelerix albiventris, Viverra civetta, Erythrocebus patas, Tragelaphus scriptus.

\section{Aponomma flavomaculatum}

La Léraba: Varanus niloticus (16MM, 8FF, 03.04.68; 6MM, 2FF, 15.02.74; O. C. C. G. E.).

Nasso (Bobo): $V$. niloticus (2MM, 2FF, 20.06.74, O. C. C. G. E.).

\section{* Bobo-Dioulasso :}

Varanus exanthematicus (10MM, 4FF, 06.05. 55 ; 12MM, 2FF, 03.05.68; O. C. C. G. E.). Python sebae (1F, 17.02.59, O. C. C. G. E.).

* Tiera (Bobo) : V. exanthematicus (3MM, 3FF, 05.04.56 ; O. C. C. G. E.).

* Koriba (Batié) : V. niloticus (2MM, 1F, 01.12 .55 , O. C. C. G. E.).

* Arli : $V$. niloticus (11MM, 7FF, 12.1959, o. C. C. G. E.).

Koro (Bobo) : V. niloticus (4MM, 5FF, $1 \mathrm{~N}$, 06.09.76, I. E. M. V. T.).

Sadina : V. niloticus (2MM, 2FF, 27.02.75, I. E. M. V. T.).

Guimpi (Kourinion) :

V. niloticus (8MM, 3FF, 15.10.76,

I. E. M. V. T.).

Naja melanoleuca (2MM, 07.01.77,

I. E. M. V. T.).

Guénako (Kourinion): V. niloticus (3MM, 1F, 2NN, 07.12.76, I. E. M. V. T.).

Kala (Gombélédougou) : V. niloticus (19MM, 11FF, 1N, 17.01.76, I. E. M. V. T.).

Diébougou: Varanus sp.: (15MM, 12FF, 10.08.75, O. C. C. G. E.).

Samogho-Iri : $V$. niloticus (8MM, 27.07.76, O. C. C. G. E.).

Riv. Lokpoho (Côte-d'Ivoire) : V. niloticus (25MM, 3FF, 2NN, 19.05.75, I. E. M. V. T.).

Lac Dalaba (Kouro) (Côte-d'Ivoire) : V. niloticus (14MM, 9FF, 28.03.75, I. E. M. V. T.).

Spécifique des varans dans les savanes de l'Atlantique jusqu'à la faille de la Rift Valley (Ethiopie, Kenya), remplacé en forêt équatoriale par Ap. arcanum; parfois sur serpents.

LAMONTELLERIE (1960) signale l'espèce de Karfiguela ; Banzo (Bobo) ; Sibi (Boromo) ; Garango, Tabsé, Koumbo, Sonbaré (Léo); Banon, Kampala (Po) ; Barbeida (Ziniaré); Zorgo. 


\section{Aponoma latum}

La Léraba : Naja melanoleuca (6MM, 2FF, 19.03 .74 , O. C. C. G. E.).

- Bobo-Dioulasso :

Python regius (3MM, 1F, 11.03.55, O. C. C. G. E.).

Python sebae (7MM, 3FF, 17.02.59, O. C. C. G. E.).

Naja nigricollis (2MM, 24.10.47; 7MM, 11FF, 14.01.63 ; O. C. C. G. E.).

* Bobo-Dioulasso :

Psammophis elegans (1M, 07.04.49, O. C. C. G. E.).

Psammophis sibilans (2FF, 02.02.76, O. C. C. G. E.).

Forêt du Kou (Nasso) : Naja nigricollis (1M, $1 F, 10.02 .75$, O. C. C. G. E.).

Toukoro: Python sebae (1N, 07.12.73, I. E. M. V. T.).

Guimpi (Kourinion): Naja melanoleuca (3MM, 2FF, 43NN, 1L, 07.01.77, I.E.M.V.T.).

Guénako (Kourinion): Naja melanoleuca (8MM, 3FF, 5NN, 1L, 22.12.76, I.E.M.V.T.).

Diébougou : Causus rhombeatus (5MM, 2FF, 2NN, 04.05.52, VILLIERS, 1955).

Spécifique des serpents dans les savanes et forêts d'Afrique au sud du Sahara ; parfois sur varans.

LAMONTELLERIE (1966) ajoute comme localités de récolte Toussiana (Bobo), FadaNgourma, Garango, et signale comme hôte supplémentaire Rhamphiophis oxyrhynchus.

\section{Aponomma transversale}

* Bobo-Dioulasso : Python regius (8MM, $5 F F, 2 N N, 11.03 .55$, O. C. C. G. E. : MOREL, 1961).

Spécifique des pythons dans toute l'Afrique.

\section{Boophilus decoloratus}

* Sabtenga (Ouagadougou) : Numida meleagris domestique $(1 \mathrm{~F}, 22.02 .56$; MOREL, 1958, B. decoloratus pp.).

Tin Akof (Dori) : Numida meleagris sauvage (1N, 19.04.63 ; LAMONTELLERIE, 1966).
B. decoloratus est normalement parasite des ongulés en Afrique. En Afrique occidentale et centrale, il n'est rencontré que sur le bétail, jamais sur les ongulés sauvages. Il en est de même avec $B$. annulatus.

Les récoltes de $B$. decoloratus sur pintades paraissent atypiques.

\section{Boophilus geigyi}

Komoé :

Alcelaphus major (2FF, 2NN, 01.01.77; 18MM, 50FF, 23.01.77, I. E. M. V. T.).

Hippotragus equinus (3MM, 1F, $1 \mathrm{~N}$, 19.02.77, I. E. M. V. T.).

Gombélédougou :

Alcelaphus major (3FF, 02.02.75,

I. E. M. V. T.).

Hippotragus equinus (5FF, 05.01.75,

I. E. M. V. T.).

C'est le seul Boophilus rencontré sur ongulés sauvages en Afrique occidentale. Il est connu du Sénégal (Bakor (Kolda)) et Niokolokoba : bubale de Luzarche; Natadji (Kédougou : guib et phacochère), du Bénin (Bori : bubale), du Niger (au nord du W : bubale, hippotrague; HOFFMANN et LINDAU, 1971), du Centrafrique (nord-est : bubale, hippotragues, éland de Derby ; THAL, 1972).

\section{Haemaphysalis hoodi}

* Bobo :

Centropus senegalensis (2MM, 20.09.55, O. C. C. G. E.).

Francolinus bicalcaratus (1N, 14.08.57, O. C. C. G. E.).

Guéna (Orodara) :

Numida meleagris (1M, 12.08.60, O.C.C.G.E.).

Francolinus bicalcaratus (1M, 2FF, 12.08.60, O. C. C. G. E.).

Coucals, francolins et pintades constituent les hôtes habituels d'Hm. hoodi dans toutes les zones d'Afrique continentale recevant au moins $1000 \mathrm{~mm}$ de pluies (savanes et forêts).

\section{Haemaphysalis houyi}

* Niamgoloko : Xerus erythropus (5MM, 11.1957, O. C. C. G. E.). 
La Léraba : X. erythropus (6FF, 06.06.75, O. C. C. G. E.).

Bobo: $X$. erythropus (7MM, 5FF, 5NN, 22.11.62, O. C. C. G. E.).

Gombélédougou : Atelerix albiventris (1M, 19.01.77, I. E. M. V. T.).

LAMONTELLERIE (1966) ajoute la référence de Dingasso (Bobo) sur $X$. erythropus (1M, 12NN).

$H m$. houyi est spécifique de l'écureuil terrestre Xerus erythropus sur toute son aire de distribution, en Afrique occidentale, centrale jusqu'au Kenya (rive est du lac Victoria), du sud du Sahel jusqu'aux savanes côtières équatoriales.

\section{Haemaphysalis leachi}

La systématique des espèces du groupe d' $\mathrm{Hm}$. leachi est actuellement en cours de révision. $\mathrm{Ce}$ sont normalement des parasites de carnivores (Canidés, Hyénidés, Viverridés, Félidés). Les résultats des études sur la systématique, la distribution et les affinités d'hôte de chaque espèce sont encore incomplètement publiés.

En Afrique occidentale et centrale, les espèces de ce groupe seront les suivantes : Hm. leachi s. str., Hm. paraleachi, Hm. punctaleachi, Hm. moreli, Hm. spinulosa (Hm. muhsamae), Hm. aethiopica.

Ces espèces peuvent se retrouver secondairement sur hérissons, lièvres, chiens; les larves et les nymphes, sur rongeurs myomorphes.

Des récoltes de ce groupe de $H m$. leachi ont été faites en Haute-Volta :

* Santidougou (Bobo) : lion (2MM, 22.09.55, O. C. C. G. E.).

Samandéni (Bobo) : panthère (1M, 21.07.57, O. C. C. G. E.).

Bobo-Dioulasso : Atelerix albiventris (4MM, 15.07.63, O. C. C. G. E.).

* Batié : Viverra civetta $(1 \mathrm{M}, 1 \mathrm{~F}, 15.01 .56$, O. C. C. G. E.).

LAMONTELLERIE (1966) mentionne des récoltes à Pama (genette), Garango (Cricetomys gambianus, larves, 04.08.62), Kontoéga (Garango, chien, civette), Dorosogua (Kongoussi : lièvre).

\section{Haemaphysalis rugosa}

* Diébougou : Ourebia ourebi $(1 \mathrm{~F}, 26.01 .47$, O. C. C. G. E.).

* Dougoumato (Kombia) : Sylvicapra grimmia $(1 \mathrm{~F}, 02.08 .56$, O. C. C. G. E.).

* Bobo : Redunca redunca (1M, 1F, 30.06.57, O. C. C. G. E.).

* Daramandougou (Banfora): bœuf (1F, 12.06.56, I. E. M. V. T.).

Ces références avaient été publiées sous le nom de Hm. aciculifer (Morel, 1958).

$\mathrm{Hm}$. rugosa parasite les ongulés et carnivores sauvages dans les savanes soudano-guinéennes et guinéennes; elle se retrouve parfois sur le bétail.

Sur animaux sauvages, elle est signalée du Sénégal (Niokolokoba : guib, radunca ; ourebi ; civette, Herpeste ichneumon), du Bénin (Bori : bubale); d Ghana (Wa:dara : redunca), de Côte-d'Ivoire (Toumodi : redunca), du Centrafrique (nord-est: bubale, THAL, 1972), du Zaïre (Haut-Uele : Kobus defassa).

\section{Ixodes aulacodi}

La Léraba: Thrynomys swinderianus (5FF, 06.1973, O. C. C. G. E.).

Parasites spécifiques de l'aulacode (avec $R h i$ picephalus simpsoni) dans les savanes soudanoguinéennes, guinéennes et en forêt équatoriale (Afrique occidentale et centrale).

\section{Hyalomma nitidum}

Dougoumato (Koumbia) : Phacochoerus aethiopicus (1M, 3FF, 16.06.58, O. C. C. G. E.).

Koumbia: Phacochoerus aethiopicus (3MM, 2FF, 03.1963, O. C. C. G. E.).

Wakara (Dédougou): Hippotragus equinus (13MM, 3FF, 03.08.62, O. C. C. G. E.).

Sara : Phacochoerus aethiopicus (2MM, 03.08.62, O. C. C. G. E.).

Kari (Houndé) : Hippotragus equinus $(1 \mathrm{~F}$, 03.03.74; 2MM, 19.07.74, O. C. C. G. E.).

Gombélédougou : Hippotragus equinus (2MM, 05.01 .75 , I. E. M. V. T.).

$H$. nitidum est parasite des ongulés domestiques et sauvages dans les savanes soudanoguinéennes et guinéennes jusqu'au nord-est du 
Centrafrique, où il a été récolté sur buffle, hippotrague, Cob de buffon et phacochère.

Il est-connu aussi du Sénégal (Kolda : phacochère; Tambakounda : phacochère) et du Bénin (Kandi et Séfou : hippotrague).

Il cohabite avec $H$. truncatum au sud de l'aire de distribution de ce dernier en Afrique occidentale et centrale.

\section{Hyalomma rufipes}

Sampelga (Dori): Quelea quelea (2NN, 13.08.62, O. C. C. G. E.).

Pont sur Volta-Blanche (route Ouaga-Kaya) : Francolinus bicalcaratus (2NN, 22.02.71, O. C. C. G. E.).

Gombélédougou : Alcelaphus major (1M, 02.02.75, I. E. M. V. T.).

Bobo-Dioulasso :

Eupodotis senegalensis (2NN, 4LL, 10.12.59, O. C. C. G. E.).

Cossypha albicapilla (1N, 07.07.74, O. C. C. G. E.).

Lepus crawshayi $(1 \mathrm{~N}, 14.02 .74$, O.C.C.G.E.).

Samogho-Iri (Orodara): Lepus sp. (2NN, 26.07.74, O. C. C. G. E.).

Soumousso (Baré) : Cinnyricinclus leucogaster (1N, 30.04.75, O. C. C. G. E.).

Samandéni: Serinus mozambicus (18NN : 13MM, 5FF, 08.05.74, O. C. C. G. E.).

Karankasso : Syncerus caffer : (1M, 05.1954, O. C. C. G. E.).

La Léraba :

Otus leucotis (1N, 1M, 04.1976, O. C. C. G.E.).

Tyto alba affinis (5NN, 13.04.76, O. C. C. G. E.).

LAMONTELLERIE (1960) signale de nombreuses localités de récoltes pour $H$. rufipes et cite comme hôtes sauvages le buffle et l'hippotrague.

H. rufipes est parasite aux stades prẻimaginaux des oiseaux et des lièvres, au stade adulte des ongulés domestiques et sauvages dans les steppes et savanes d'Afrique au sud du Sahara.

\section{Hyalomma truncatum}

Dori : Phacochoerus aethiopicus (1F, 17.02.62, O. C. C. G. E.).
- Sabtenga (Boussé) : Tragelaphus scriptus (2MM, 3FF, 19.02.56, I. E. M. V. T.).

- Houndé: Hippotragus equinus (24MM, 4FF, 16.06.39, O. C. C. G. E.).

- Koumbia : Phacochoerus aethiopicus (1M, 1F, 03.03.63, O. C. C. G. E.).

Wakara (Dédougou): Hippotragus equinus (1F, 03.08.62, O. C. C. G. E.).

* Bobo-Dioulasso : Lepus crawshayi (4NN, $03.12 .56 ; 15 \mathrm{NN}, 08.12 .58 ; 8 \mathrm{NN}, 14.02 .74$, O. C. C. G. E.).

Karankasso: Syncerus caffer (1M, 05.1954, O. C. C. G. E.).

* Diébougou : Hippotragus equinus (7MM, 3FF, 21.03.57, O. C. C. G. E.).

Batié : Phacochoerus aethiopicus (7MM, 10FF, 04.1959, O. C. C. G. E.).

Komoé : Phacochoerus aethiopicus (2MM, 23.01.77; 1M, 06.02.77, J. E. M. V. T.).

LAMONTELLERIE (1966) donne de nombreuses localités de récoltes et cite le lion comme hôte sauvage supplémentaire pour la HauteVolta.

Les adultes de $H$. truncatum parasitent les ongulés domestiques et sauvages (parfois carnivores) au sud du Sahel, dans les savanes soudaniennes et soudano-guinéennes. Les larves et les nymphes se gorgent ordinairement sur rongeurs Myomorphes, parfois sur lièvres. L'espèce répandue dans toute l'Afrique au sud du Sahara.

\section{Rhipicephalus cuspidatus}

Cette espèce est inféodée aux terriers d'oryctéropes, le plus souvent occupés par des phacochères, et qui servent de gîte temporaire à d'autres espèces de mammifères ou d'oiseaux, en Afrique occidentale et centrale.

L'hôte le plus fréquent de tous les stades est Phacochoerus aethiopicus. Les récoltes citées ci-après, sauf indication spéciale, ont toutes été faites sur cet hôte.

Dori (3MM, 1F, 17.02.62, O. C. C. G. E.).

Fada-Ngourma (2MM, 6FF, 94NN, 155LL, 18.02.74, O. C. C. G. E.).

Nabéré (Gombélédougou) : Thryonomys swinderianus (2FF, $11 \mathrm{NN}, 24.03 .74$, O. C. C. G. E.).

Koumbia :(6NN, 5FF, 03.1963, O.C.C.G.E.). 
* Pé (Koumbia) : (1F, 06.1949, O. C. C. G. E.).

* Dougoumato (Koumbia) :

Phacochoerus aethiopicus (1F, 19.06.59, O. C. C. G. E.).

Hystrix cristata (14NN, 20.03.57, O.C. C. G. E.).

Sara : (20MM, 18FF, 03.08.60, O.C.C.G. E.).

* Sono (Nouna): (8MM, 4FF, 13.02.59, O. C. C. G. E.).

Komoé : (5MM, 25.01.76:15MM, 27FF, $2 \mathrm{NN}, 23.01 .77 ; 1 \mathrm{MM}, 7 \mathrm{FF}, 06.02 .77 ; 11 \mathrm{MM}$, $1 F, 26.02 .77$, I. E. M. V. T.).

La Léraba : (8MM, 12FF, 30.05.68, O. C. C. G. E.).

LAMONTELLERIE (1960) cite cette tique de Fada-Ngourma, Madiagouné (Fada), Tabsé (Garango), Manga, Po, Kampala (Po), Dimnogoué (Ziniaré), Bonboré (Zorgo), $12^{\circ} 10 \mathrm{~N}-0^{\circ} 5 \mathrm{~W}$ (Zorgo, Hyaena hyaena, MM, FF, NN). Hors de Haute-Volta, des adultes ont été également récoltés sur Crocuta crocuta, Hyaena hyaena, des nymphes et des larves sur Canis aureus, Mungos mungo, Herpestes ichneumon, Ichneumia albicauda, Felis serval, Panthera pardus, Ourebia ourebi, Gazella rufifrons, Francolinus bicalcaratus.

\section{Rhipicephalus guilhoni}

* Koumbara (Tougan): Lepus sp. (1M, 4FF, 19.10.54, O. C. C. G. E.).

* Kotédougou (Bobo): Neotis caffra (1M, 27.06 .55 , O. C. C. G. E.).

* Ouakuy (Houndé) : Hippotragus equinus (4MM, 1F, 20.08.49, O. C. C. G. E.).

* Pé (Koumbia) : Phacochoerus aethiopicus (5MM, 4FF, 06.1949, O. C. C. G. E.).

Les adultes de $R h$. guilhoni sont parasites des ongulés et carnivores, domestiques ou sauvages, dans les steppes sud-sahéliennes et dans les savanes soudaniennes. Larves et nymphes se gorgent sur rongeurs myomorphes.

A la suite des références de récoltes en HauteVolta, LAMONTELLERIE (1966) cite comme hôtes sauvages supplémentaires le lion et Eupodotis senegalensis.

\section{Rhipicephalus lunulatus}

Badema (Bobo) : Hystrix cristata (1M, 11. 11.60, O. C. C. G. E.).

Rh. lunulatus habite les savanes humides d'Afrique occidentale, centrale et orientale. Il n'est jamais très abondant. Il peut parasiter toutes les espèces disponibles d'ongulés et de carnivores, domestiques ou sauvages. La récolte sur porc-épic semble rare. Les larves et les nymphes sont associées aux rongeurs myomorphes.

\section{Rhipicephalus muhsamae}

Gombélédougou : Hippotragus equinus (1M* 05.01.75, I. E. M. V. T.).

* Koumbia : Thryonomys swinderianus (2FF, 03.1963 , O. C. C. G. E.).

Nabéré (Diébougou): Thryonomys swinderianus $(1 \mathrm{~F}, 24.03 .74$, O. C. C. G. E.).

* Dougoumato (Koumbia) : Phacochoerus aethiopicus (2MM, 19.06.59, O. C. C. G. E.).

* Samandeni (Bobo) : Panthera pardus (4MM, 1F, 21.07.57, O. C. C. G. E.).

* Santidougou (Bobo) : Panthera leo (4MM, 5FF, 22.09.55, O. C. C. G. E.).

* Badéma (Bobo) : Hystrix cristata (3MM, $1 F, 11.11 .60$, O. C. C. G. E.).

LAMONTELLERIE (1966) cite ces mêmes hôtes sauvages sous le nom de $R h$. simus simus.

La distribution de $R h$. muhsamae comprend le sud du Sahel, les savanes soudaniennes et les zones ouvertes et sèches des savanes soudanoguinéennes et oubanguiennes, et s'étend jusqu'au Rwanda et à la rive occidentale du lac Victoria.

Les hôtes des adultes sont pratiquement tous les ongulés et carnivores, domestiques et sauvages, ainsi, que l'aulacode et le porc-épic, disponibles dans l'aire de distribution de l'espèce. Les préimagos parasitent les rongeurs myomorphes.

\section{Rhipicephalus senegalensis}

La Léraba : Phacochoerus aethiopicus (MM, FF, 30.05.68, O. C. C. G. E.).

Komoé : Syncerus caffer (5MM, 1F, 02.01.77, I. E. M. V. T.). 
Diébougou : Ourebia ourebi $(1 \mathrm{~F}, 26.01 .47$, O. C. C. G. E.).

* Pé (Koumbia) : Phacochoerus aethiopicus (8MM, 4FF, 06.1949, O. C. C. G. E.).

Dougoumato (Koumbia) :

Phacochoerus aethiopicus (9MM, 8FF, 04. 1959, O. C. C. G. E.).

Sylvicapra grimmia (2MM, 16.06.58, O. C. C. G. E.).

Hystrix cristata (1F, 20.03.57, O. C. C. G. E.).

Sara : Phacochoerus aethiopicus (17MM, 13FF, 03.08.60, O. C. C. G. E.).

Wakara (Dédougou) : Panthera pardus (1F, 23.02.63, O. C. C. G. E.).

La distribution de $R h$. senegalensis correspond aux savanes guinéennes, oubanguiennes et soudano-guinéennes. Les adultes y sont potentiellement parasites de tous les ongulés et carnivores domestiques ou sauvages, ainsi que du porc-épic.

Les savanes soudano-guinéennes, qui sont bien représentées à l'ouest de la Haute-Volta, constituent la zone de coexistence de $R h$. senegalensis et de Rh. muhsamae; leurs hôtes sont les mêmes. Il semble que $R h$. senegalensis soit normalement inféodé à la végétation de basfonds et à la savane boisée, tandis que $R h$. muhsamae, moins hygrophilc, serait plutôt associé aux pentes, aux plateaux, à la savane ouverte.

Les hôtes des préimagos sont les rongeurs myomorphes.

\section{Rhipicephalus simpsoni}

La Léraba: Thryonomys swinderianus (1M, 2FF, 06.1973; 2MM, 5NN, 14.04.74;1M, 17.02.74, O. C. C. G. E.).

Nabéré (Gombélédougou) : Thryonomys swinderianus $(1 \mathrm{M}, 23.03 .74$, O. C. C. G. E.).

Boroum (Gaoua) : Thr. swinderianus (3MM, 13.02.62, O. C. C. G. E.).

Batié : Thr. swinderianus (2MM, 1F, 25.02.62, O. C. C. G. E.).

Parasite spécifique à tous les stades de l'aulacode, sur toute l'aire de distribution de l'hôte en Afrique occidentale, centrale et orientale.

\section{Rhipicephalus sulcatus}

La Léraba:

Ourebia ourebi $(1 \mathrm{~F}, 07.06 .68$, O. C. C. G. E.).

Lepus crawshayi (2MM, 3FF, 07.06.68; 3MM, 3FF, 18.04.73, O. C. C. G. E.).

Komoé :

Ourebia ourebi (1F, 05.12.76, I. E. M. V. T.). Alcelaphus major (2MM, 05.02.77, I. E. M. V. T.).

Phacochoerus aethiopicus (3MM, 4FF, 23.01. 77, I. E. M. V. T.).

* Toussiana : Atelerix albiventris (2MM, 2FF, 20.08.77, O. C. C. G. E.).

* Karankasso : Lepus crawshayi (11MM, 6FF, 26.04.57, O. C. C. G. E.).

* Dougoumato (Koumbia) :

Lepus craw'shayi (2MM, 1F, 16.06.68, O. C. C. G. E.).

Sylvicapra grimmia (7MM, 4FF, 02.08.56; 2MM, 5FF, 16.06.58, O. C. C. G. E.).

* Sinorosso (Baré) : Lepus crawshayi (3MM, 1F, 11.10.57, O. C. C. G. E.).

Gombélédougou :

Alcelaphus major (1F, 02.02.75, I.E.M.V.T.).

Hippotragus equinus (2MM, 05.01.75, I. E. M. V. T.).

Kari (Houndé) :

Hippotragus equinus (10MM, 8FF, 19.07.74, O. C. C. G. E.).

Canis aureus (5MM, 14.02.74, O. C. C. G. E.).

Samandeni (Bobo) : Panthera pardus (3MM, 1F, 21.07.57, O. C. C. G. E.).

* Bobo-Dioulasso :

Atelerix albiventris (5MM, 05.11.56;10MM, 6FF, $15.08 .58 ; 1 \mathrm{~F}, 13.01 .76$, O. C. C. G. E. ; 12MM, 6FF, 22.07.73, I. E. M. V. T.).

Lepus crawshayi (MM, FF, 20.06.54; 16. $09.55,25.08 .56,17.11 .57 ; 1 \mathrm{~F}, 18.02 .74$; 4MM, 11FF, 06.10.75, O. C. C. G. E.).

Erythrocebus patas (8MM, 5FF, 24.08.53; $8 \mathrm{MM}, 7 \mathrm{FF}, 23.07 .57$, O. C. C. G. E.).

Sylvicapra grimmia (4FF, 06.1953;2MM, 1F, $05.07 .56 ; 4 \mathrm{MM}, 5 \mathrm{FF}, 25.08 .56 ; 11 \mathrm{MM}$, $9 \mathrm{FF}, 20.02 .57$, O. C. C. G. E.). 
* Santidougou (Bobo) : Panthera leo (5MM, 3FF, 22.09.55, O. C. C. G. E.).

* Badéma (Bobo): Lepus crawshayi (1F, 06.07 .60 , O. C. C. G. E.).

* Moami (Bobo): Lepus crawshayi (1M, 11.11.60, O. C. C. G. E.).

* Samogho-Iri (Orodara) : Lepus crawshayi (7MM, 1F, 26.07.74, O. C. C. G. E.).

* Wakara (Dédougou) : Hippotragus equinus $(1 F, 23.02 .62$, O. C. C. G. E. $)$.

LAMONTELLERIE (1966) mentionne comme hôtes supplémentaires le bubale et l'écureuil terrestre.

Les adultes de $R$. sulcatus peuvent parasiter pratiquement tous les ongulés et carnivores domestiques ou sauvages, ainsi que léporides, hérissons, singes, de même que certains grands oiseaux comme les outardes ou les autruches, dans les savanes subtropicales et subéquatoriales d'Afrique occidentale, centrale et orientale; il peut même être localement abondant dans les bas-fonds riverains au milieu des steppes masai. Ses larves et nymphes évoluent sur rongeurs myomorphes.

\section{Argas arboreus}

Argas arboreus est associé aux Ardéiformes sur tout le continent africain. Il est surtout fréquent dans les nicheries du garde-bœufs Bubulcus ibis, qui se situent ordinairement sur des arbres à proximité de zones d'inondation importantes. Il est très facile à récolter sous l'écorce des arbres qui portent les nids (très souvent Acacia albida). Il est signalé à LamordéTorodi, au Niger, près de la frontière voltaīque. Argas arboreus devrait donc être cherché dans le Sahel voltaĩque, en relation avec les hôtes les plus fréquents qui sont Bubulcus ibis, Ardea cinerea, Balearica pavonina, Leptoptilos crumenifer, Pelecanus rufescens.

\section{Argas hermanni}

Argas hermanni est un argas d'oiseaux sauvages qui se retrouve souvent dans les poulaillers et pigeonniers (signalé en Haute-Volta) en Afrique occidentale, centrale, orientale et en Egypte.

Les hôtes sauvages connus sont Francolinus bicalcaratus (Haute-Volta, Santidougou, nom- breuses larves, 25.05.60, LAMONTELLERIE, 1966), Tyto alba affinis (Sénégal: Kolda, 03.04.64), Columba livia sauvage (Mauritanie : Aioun el Atrous, 22.11.59).

\section{Carios boueti}

Parasite des chauves-souris insectivores en Afrique, au Proche-Orient, en Asie centrale soviétique, jusqu'en Malaisie, il peut être trouvé dans les grottes ou les vieilles constructions qui servent d'abri à ses hôtes.

Il n'est pas signalé en Haute-Volta, mais doit s'y trouver. On le connaît de Missirikoro (Sikasso, Mali, LAMONTELLERIE, 1960) et de Kombo (Dapango, Togo, I. E. M. V. T.), 2 localités très proches de la frontière voltaïque.

\section{Alectorobius erraticus sonrai}

Cet ornithodore est connu de plusieurs localités dans les steppes sahéliennes ou dans les savanes soudaniennes (Mauritanie, Sénégal, Mali, Niger, Tchad). Il a toutes chances de se retrouver en Haute-Volta dans ces mêmes zones, dans les terriers de rongeurs myomorphes, de Xerus erythropus ou de Vulpes pallida.

\section{CONCLUSIONS}

Environ 25 espèces de tiques sont connues comme parasites d'animaux sauvages en HauteVolta. Les hôtes correspondent à la faune ordinairement rencontrée. Les résultats en sont analogues à ceux observés dans les zones équivalentes (soudanienne et soudano-guinéenne) des pays voisins.

En ce qui concerne la distribution des lieux de récoltes, il faut constater qu'ils se situent pour la majeure part dans l'ouest du pays ; très peu se placent à l'est, et surtout au nord, au Sahel. Des récoltes dans cette zone comporteraient surtout des Hyalomma (H. impeltatum, $H$. rufipes, $H$. truncatum, $H$. impressum) et des Rhipicephalus (Rh. cuspidatus, Rh. guilhoni, $R h$. muhsamae), sur phacochères et surtout sur Gazella rufifrons qui manque dans les références d'hôtes en Haute-Volta. Des enquêtes sur les nombreuses espèces d'oiseaux du Sahel montreraient certainement leur rôle dans le cycle évolutif de $H$. rufipes, une des tiques les plus abondantes sur le bétail à ces latitudes. 
Il serait certainement possible d'augmenter le nombre des données en plaçant des collecteurs dans les postes de contrôle du Service des Eaux et Forêts, les dimanches de la saison d'ouverture de la chasse.

Plus particulières sont les lacunes concernant les parasites de certaines espèces à écologie très spécialisée ou intégralement protégées.

$\mathrm{Si}$ le daman de rochers (Procavia ruficeps) existe en Haute-Volta, il est certainement porteur de Rhipicephalus boueti qui en est spécifique (connu de Guinée, du Mali, du Bénin, du Cameroun).

Bien que le buffle ne soit pas rare, les références de tiques sur cet hôte sont minimes (2 récoltes) pour l'ouest du pays, nulles pour le centre et l'est. Cela est d'autant plus dommage que le buffle héberge 2 tiques spécifiques, Amblyomma splendidum et Rhipicephalus cliffordi; les 2 espèces sont connues de Côte-d'Ivoire, A. splendidum, du centre du Bénin; il serait intéressant de vérifier si les 2 tiques accompagnent leur hôte dans le nord de son aire de distribution.

Il en est de même avec l'éléphant, qui présente 2 tiques spécifiques Amblyomma tholloni et Amblyocentor circumguttatus. La première est connue seulement par une référence sur homme en ce qui concerne la Haute-Volta. Bien que la chasse à l'éléphant soit fermée, il devrait être possible de faire effectuer des prélèvements lors de battues organisées par les Eaux et Forêts lorsque des troupeaux locaux deviennent trop nombreux et nuisent aux cultures.

\section{SUMMARY}

\section{Wild animal ticks in Upper-Volta}

Twenty four species of ticks have been checked on wild animals in UpperVolta. Five to ten species more will certainly been listed, after the systematic study of the Haemaphysalis leachi group, or after tick collecting off elephant, buffalo, from nests of Ardetdae and Ciconiidae birds or burrows of small mammals.

\section{RESUMEN}

Garrapatas de animales salvajes en Alto-Volta

Veinte cuatro especies of garrapatas resultan conocidas en los animales salvajes del Alto-Volta. Se añadiran probablemente de 5 a 10 especies más, después del estudio sistemático del grupo de la Haemaphysalis leachi o de colectas de garrapatas en el elefante, en el bufalo, en los nidos de las aves Ardeidae y Ciconiidae, o en las madrigueras de los roedores.

\section{BIBLIOGRAPHIE}

1. AESCHLimANN (A.). Biologie et écologie des tiques (Ixodoidea) de Côte-d'Ivoire. Acta trop., 1967, 24 (4) : 282-405.

2. HOFFMANN (G.), LINDAU (M.). Zecken an Nutz- und Wildtieren in Niger. $Z$. angew. Entom., 1971,69 (1) : 72-82.

3. HOOGSTRAAL (H.) (1956). African Ixodoidea. I. Ticks of the Sudan. Washington, U. S. Govt print. Office, 1956.1100 p. (Rev. Rép. N. A. M. R. U., $n^{0}$ 3, 005-050-29-07).

4. MOREL (P. C.). Tiques d'animaux sauvages. Le parc national du Niokolo-Koba, Sénégal. I. Mém. Inst. fr. Afr. noire, 1956, $48: 229-232$.

5. MOREL (P. C.). Rhipicephalus boueti n. sp. (Acarina, Ixodidae) parasite des damans du Dahomey. Bull. Soc, Path. exot., 1957, 50 (5) : 696-700.

6. MOREL (P. C.). Les tiques des animaux domestiques de l'Afrique occidentale française. Rev. Elev. Méd. vét. Pays trop., 1958, 11 (2) : 153-189.

7. MOREL (P. C.). Tiques (Acarina Ixodoidea) Deuxième note. Le parc national du Niokolo-Koba (Sénégal). II. Mém. Insí. fr. Afr. noire, 1961, 62 : 83-90.

8. MOREL (P. C.). Tiques (Acarina Ixodoidea). La réserve naturelle intégrale du Mont Nimba. V. Mém. Inst. fr. Afr. noire, $1963,66: 33-40$.

9. MOREL (P. C.), FINELLE (P.). Les tiques des animaux domestiques du Centre Afrique. Rev. Elev. Méd. vét. Pays trop., 1961, 14 (2) : 191-197.

10. MOREL (P. C.), GRABER (M.). Les tiques des animaux domestiques du Tchad. Rev. Elev. Méd. vét. Pays trop., 1961, 14 (2) : 199-203.
11. MOREL (P. C.), MAGIMEL (J.). Les tiques des animaux domestiques de la région de Fort-Lamy (Tchad) et Fort-Foureau (Cameroun). Rev. Elev. Méd. vét. Pays trop., 1959, 12 (1) : 53-57.

12. MOREL (P. C.), MOUCHET (J.). Les tiques du Cameroun (Ixodidae et Argasidae). Annls Parasit. hum. comp., 1958, 33 (1-2) : 69-111.

13. MOREL (P. C.), MOUCHET (J.). Les tiques du Cameroun (Ixodidae et Argasidae). II. Annls Parasit. hum. comp., 1965, 40 (4) : 477-496.

14. MOREL (P. C.), VASSILIADES (G.). Les Rhipicephalus du groupe sanguineus: espèces africaines (Acariens, Lxodidae). Rev. Elev. Méd. vét. Pays trop., 1963, 15 (4) : 343-386.

15. MOREL (P. C.), VASSILIADES (G.). Description de Rhipicephalus muhsamae n. sp. de l'Ouest Africain (groupe de $R h$. simus; Acariens, Ixodoidea). Rev. Elev. Méd. vét. Pays trop., 1964, 17 : 619-636.

16. ROUSSELOT (R.). Ixodes de l'Afrique noire. Bull. Soc. Path. exot., 1951, 44 (5-6) : 307-309.

17. ROUSSELOT (R.). Notes de parasitologie. II. Ixodes. Paris, Vigot, 1953. 135 p.

18. THAL (J. A.). Les maladies similaires à la peste: étude et lutte. Ndélé, République Centrafricaine. Projet FAO/PNUD CAF 13. Rapport final. MaisonsAlfort, I. E. M. V. T., 1972, 37 p., 40 tabl., 3 cartes, 29 photogr.

19. VILLIERS (A.). Note sur quelques Ixodidae et Gamasidae, parasites des vertébrés rencontrés en Afrique occidentale française. Bull. Inst. fr. Afr. noire, $1955,17 \mathrm{~A}: 444-454$. 